\title{
Minimum Codebook Size to achieve Maximal Diversity Order for RVQ based MIMO Systems
}

\author{
Daniel Castanheira, Adão Silva, Atílio Gameiro \\ DETI, Instituto de Telecomunicações, University of Aveiro, \\ Emails: dcastanheira@av.it.pt, asilva@av.it.pt, amg@ua.pt
}

\begin{abstract}
Multiple antenna systems provide both capacity and diversity gains relatively to single antenna based networks. These gains rely heavily on the availability of channel state information. In this manuscript we assume that the receiver has accurate channel information, which is quantized and fed back to the transmitter. Sufficient conditions for the feedback rate are provided that guarantee no loss of diversity due to channel quantization. Considering a $K \times M$ MIMO system, we show that to achieve a diversity of $D=M-K+1$ which is the maximum allowable, a feedback channel with rate of $\log _{2}(1+D)$ bits per channel use is enough.
\end{abstract}

Index Terms-Zero-Forcing, MIMO Systems, Diversity Methods, Rayleigh Channels

\section{INTRODUCTION}

It is widely known that for a multiple-input multiple-output (MIMO) system the capacity scales linearly with the minimum number of transmit and receive antennas [1]. Furthermore, MIMO systems provide diversity advantages beyond capacity improvements. However, there is a fundamental tradeoff between the achievable diversity and capacity [2]. Spatial multiplexing, with the help of linear receivers and transmitters is a low complexity solution that allows to obtain the aforementioned benefits.

The availability of channel state information (CSI) at the transmitter enables the adaptation of the transmitted signal to the current channel conditions. On the opposite side the lack of CSI limits considerably the performance both in terms of diversity achieved and signal to noise ratio (SNR) [3]. CSI can be acquired using the channel reciprocity property in time division duplex (TDD) systems, or through a feedback channel from the receiver to the transmitter. In a feedback system the receiver quantizes the channel using a codebook known to both terminals, and feeds back the selected index to the transmitter. A too coarse quantization implies that the CSI available may be too limited to perform an accurate channel adaptation whereas if it is very precise this implies strong overhead for signaling. It is therefore important to quantify how the relevant link metrics in a MIMO system are affected by a limited feedback. The topic of limited feedback has been addressed in several publications. In [4] the authors provide a concise characterization of the gain with each additional bit of feedback information. In addition, it is observed a relationship between the quantized beamforming and Grassmannian line packing problems [5]. Other codebook design methods have been proposed as alternatives to Grassmannian line packings. Namely, ran- dom vector quantization (RVQ) [6], fourier codebooks [7] and equiangular frame based codebooks [8], just to name a few.

The capacity and the probability of symbol error (SER) are two common measures used to measure the performance of a codebook. The array gain and the diversity order are enough to parameterize the probability of error, in the high SNR regime. For the case of single stream beamforming, in [9] necessary and sufficient conditions for the sets of possible beamformers and combiners are derived that guarantee full diversity order. In [10] a tight SER upper bound is derived. Still for the single stream case, in [11] the authors analyse the impact of delay and feedback errors in the system performance. For spatial multiplexing MIMO systems with linear receiver structure, it was demonstrated in [12] that transmit antenna selection achieves the maximum diversity. Nevertheless, as shown in [3] this is not the most efficient scheme in terms of feedback signaling and maximum diversity can still be achieved with less feedback. Namely, the authors demonstrate that maximum diversity is guaranteed if the codebook size is greater or equal than $K(M-K)+1$, where $M$ and $K$ are the number of transmit and receive antennas, respectively. In this manuscript, an improved result is obtained by showing that a codebook with size $M-K+2$ is enough to achieve maximum diversity. When $M$ is much larger than $K$ this leads to a codebook with a size $K$ times lower (approximately) or equivalently to a reduction in the feedback rate of approximately $\log _{2}(K)$ bits. More specifically, we derive an upper bound for the average bit error rate (BER) and provide sufficient conditions for the codebook size that guarantee maximum diversity. Simulation results of the average BER for a $K \times M$ MIMO system verify our claim.

The rest of this paper is organized as follows: Section II introduces the system model. In section III we derive sufficient conditions to achieve maximum diversity. Then, in Section IV we provide a numerical confirmation of the derived sufficient conditions. Finally, section V provides some conclusions.

Notations: Boldface capital letters denote matrices, boldface lowercase letters denote column vectors. The operations $(.)^{H}$ and $\operatorname{tr}($.$) represent the Hermitian transpose and the$ trace of a matrix. $\mathcal{N}_{R}[\mathbf{A}]$ denote the right null-space of matrix A. The notation $\mathbf{A} \sim \mathbf{B}$ denotes that matrix $\mathbf{A}$ and $\mathbf{B}$ are identically distributed. $\lambda_{m}[\mathbf{A}]$ denotes the $m$ th largest singular value of matrix $\mathbf{A}$ and $|\mathbf{A}|$ the matrix $\mathbf{A}$ determinant. For two functions $f(x)$ and $g(x), f(x) \dot{\leq} g(x)$ means $\lim _{x \rightarrow \infty} f(x) \leq \lim _{x \rightarrow \infty} g(x)$ [13]. 


\section{System MODEL}

We consider transmission from a single base-station (BS), equipped with $M$ antennas, to a mobile terminal (MT) with $K$ antennas, as shown in Fig. 1. The BS uses all available channel dimensions $(\min (M, K))$, i.e. it transmits $K$ data streams. Both terminals use linear processing techniques. Namely, the BS uses a precoder $\mathbf{Q}$ and the $\mathrm{MT}$ an equalizer $\mathbf{W}$. For the design of the precoder and equalizer it is assumed full channel state information at the receiver (CSIR) and no channel state information at the transmitter (CSIT). The only channel information available at the transmitter is obtained through a low-rate feedback link from the receiver, see Fig. 1. Namely, the precoder $\mathbf{Q}$ is selected from a finite set $\mathcal{T}=\left\{\mathbf{Q}_{1}, \ldots, \mathbf{Q}_{T}\right\}$, with cardinality $T$, at the receiver end and then the corresponding index is feedback to the transmitter. This set is assumed to be fixed and known by both terminals. To select the best codeword from set $\mathcal{T}$ the receiver uses as a metric the system BER. In the following, we assume that set $\mathcal{T}$ is generated using the random vector quantization (RVQ) method [14].

The transmit signal $\left(x \in \mathbb{C}^{M \times 1}\right)$ is given by

$$
\mathbf{x}=P^{\frac{1}{2}} \mathbf{Q d}
$$

where $\mathbf{Q} \in \mathbb{C}^{M \times K}, \mathbf{d} \in \mathbb{C}^{K \times 1}$ and $P$ denote respectively the transmit precoder, the vector of data symbols (drawn from a QAM constellation) and the overall average transmit power of the BS.

The received signal at the MT can be expressed by

$$
\mathbf{y}=\mathbf{H x}+\mathbf{n}
$$

where $\mathbf{H}$ and $\mathbf{n}$ denote the channel between BS and MT and zero mean white Gaussian noise with variance $\sigma^{2}$, respectively. In the following it is assumed that the entries of the channel matrix $\mathbf{H}$ are independent and complex-Gaussian distributed.

To decode the transmitted symbols the MT applies the equalizer $\mathbf{W} \in \mathbb{C}^{K \times K}$ to the received signal to obtain $\mathbf{r} \in \mathbb{C}^{K \times 1}$

$$
\mathbf{r}=\mathbf{W y}=\mathbf{W H x}+\mathbf{W n}
$$

In the following we focus on the zero-forcing equalizer. According to [3] we have that the SNR of the $k$ th stream is

$$
\rho_{k}(\mathbf{Q})=\frac{P}{\sigma^{2}}\left(\left[\mathbf{Q}^{H} \mathbf{H}^{H} \mathbf{H Q}\right]_{k k}\right)^{-1}
$$

By taking into account the precoder selection process the system BER, for QPSK, is as follows

$$
\overline{B E R}(P, \mathcal{T})=\mathbb{E}_{\mathbf{H}}\left[\min _{\mathbf{Q} \in \mathcal{T}} \frac{1}{K} \sum_{k=1}^{K} \mathcal{Q}\left(\sqrt{2 \rho_{k}(\mathbf{Q})}\right)\right]
$$

where $\mathcal{Q}(x)=1 / \sqrt{2 \pi} \int_{x}^{+\infty} e^{-t^{2} / 2} d t$ is the Q-function.

\section{ANALYSIS}

In this section we derive sufficient conditions for codebook $\mathcal{T}$ that guarantee no loss in diversity due to quantization. Namely, we show that to achieve diversity order of $D=M-K+1$ we need a RVQ codebook with size $1+D$.

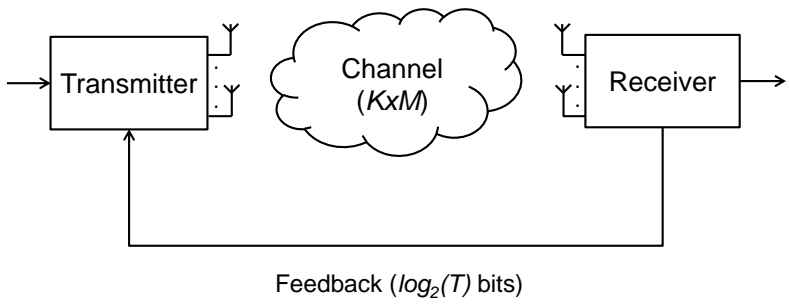

Fig. 1. Block diagram of the considered system.

The use of multiple antennas at the transmitter and/or receiver provides multiple paths to the information signal to pass through. This provides multiple independently faded replicas of the data symbol, at the receiver end, hence more reliable reception is achieved [13]. Intuitively the diversity order is equal to the number of independent channel paths the information signal passes through [13]. To formalize it we have the following definition [13]: a system achieves a diversity $c$ if its BER follows

$$
\lim _{P \rightarrow \infty}-\frac{\log (\overline{B E R}(P, \mathcal{T}))}{\log (P)}=c
$$

It is well known that for a $M \times K$ MIMO system, with $K$ data streams, the diversity order is equal to $M-K+1$ [15]. This is achieved with CSIT, in the following we will show that the same diversity is achievable when only limited channel information is available at the transmitter.

The ZF equalizer removes completely the inter-stream interference. Namely, to recover the $k$ th stream the received signal is multiplied by vector $\mathbf{w}_{k}$ which is in the null-space of the other $K-1$ streams

$$
\mathbf{w}_{k}=\mathcal{N}_{R}\left[\mathbf{H} \hat{\mathbf{Q}}_{k}\right] .
$$

where $\hat{\mathbf{Q}}_{k}=\left[\mathbf{Q}_{1}, \mathbf{Q}_{k-1}, \mathbf{Q}_{k+1}, \ldots, \mathbf{Q}_{K}\right]$. Let us assume that $\mathbf{w}_{k}$ is a unitary vector, i.e $\mathbf{w}_{k}^{H} \mathbf{w}_{k}=1$, as multiplication by a scalar does not affect the resulting stream SNR. The received signal after the application of filter $\mathbf{w}_{k}$ is given by $\mathbf{w}_{k}^{\mathbf{H}} \mathbf{H Q}$ and the respective $\mathrm{SNR}$ is

$$
\rho_{k}(\mathbf{Q})=\frac{P}{\sigma^{2}} \mathbf{w}_{k}^{H} \mathbf{H} \mathbf{Q} \mathbf{Q}^{H} \mathbf{H}^{H} \mathbf{w}_{k}
$$

As (8) is a Rayleigh quotient the SNR of the $k$ th stream is bounded by

$$
\frac{P}{\sigma^{2}} \lambda_{K}\left[\mathbf{H Q Q} \mathbf{Q}^{H} \mathbf{H}^{H}\right] \leq \rho_{k}(\mathbf{Q}) \leq \frac{P}{\sigma^{2}} \lambda_{1}\left[\mathbf{H Q Q} \mathbf{Q}^{H} \mathbf{H}^{H}\right]
$$

where $\lambda_{K} \leq \ldots \leq \lambda_{1}$ represent the ordered nonzero singular values of matrix $\mathbf{H Q Q} \mathbf{Q}^{H} \mathbf{H}^{H}$ which are equal to the nonzero singular values of matrix $\mathbf{Q}^{H} \mathbf{H}^{H} \mathbf{H Q}$.

Consider the singular value decomposition of channel $\mathbf{H}=$ $\mathbf{U D V}{ }^{H}$, where $\mathbf{D}$ is a rectangular diagonal matrix containing the matrix $\mathbf{H}$ singular values. Form a channel $\mathbf{G}$ with all nonzero singular values equal to the worst singular value of $\mathbf{H}$, i.e. $\mathbf{G}=\lambda_{K}[\mathbf{H}] \mathbf{U} \mathbf{D}_{0} \mathbf{V}^{H}$, where $\mathbf{D}_{0}=[\mathbf{I}, \mathbf{0}]$. As the singular values of matrix $\mathbf{G}$ are worse than $\mathbf{H}$ we have [16]

$$
\lambda_{K}\left[\mathbf{Q}^{H} \mathbf{G}^{H} \mathbf{G Q}\right] \leq \lambda_{k}\left[\mathbf{Q}^{H} \mathbf{H}^{H} \mathbf{H Q}\right], k \in\{1, \ldots, K\}
$$


Therefore the performance under channel $\mathbf{G}$ is always worse than the one obtained with $\mathbf{H}$

$\overline{B E R}(P, \mathcal{T})$

$$
\leq \mathbb{E}_{\mathbf{G}}\left[\min _{\mathbf{Q} \in \mathcal{T}} \mathcal{Q}\left(\sqrt{\frac{2 P}{\sigma^{2}} \lambda_{K}\left[\mathbf{Q}^{H} \mathbf{G}^{H} \mathbf{G Q}\right]}\right)\right]
$$

As the Q-function is monotonic decreasing we have

$\overline{B E R}(P, \mathcal{T})$

$$
\leq \mathbb{E}_{\mathbf{G}}\left[\mathcal{Q}\left(\sqrt{\frac{2 P}{\sigma^{2}} \max _{\mathbf{Q} \in \mathcal{T}} \lambda_{K}\left[\mathbf{Q}^{H} \mathbf{G}^{H} \mathbf{G Q}\right]}\right)\right]
$$

Using the RVQ quantizer the precoder set $\mathcal{T}$ is updated at each channel realization, with seeds known at both terminals. Namely, for RVQ [6], the matrices $\mathbf{Q}_{t}, t=1, \ldots, T$ are independent and isotropic and respect $\mathbf{Q}_{t}^{H} \mathbf{Q}_{t}=\mathbf{I}$. The average BER, over the channel and precoder set, is given by

$\overline{B E R}(P)$

$$
\leq \mathbb{E}_{\mathbf{G}, \mathcal{T}}\left[\mathcal{Q}\left(\sqrt{\frac{2 P}{\sigma^{2}} \max _{\mathbf{Q} \in \mathcal{T}} \lambda_{K}\left[\mathbf{Q}^{H} \mathbf{G}^{H} \mathbf{G Q}\right]}\right)\right]
$$

As matrices $\mathbf{Q}_{1}, \ldots, \mathbf{Q}_{T}$ are isotropic then

$$
\begin{aligned}
\mathbf{Q}_{t}^{H} \mathbf{G}^{H} \mathbf{G} \mathbf{Q}_{t} & =\lambda_{K}^{2}[\mathbf{H}] \mathbf{Q}_{t}^{H} \mathbf{V} \mathbf{D}_{0}^{H} \mathbf{D}_{0} \mathbf{V}^{H} \mathbf{Q}_{t} \\
& \sim \lambda_{K}^{2}[\mathbf{H}] \mathbf{Q}_{t}^{H} \mathbf{D}_{0}^{H} \mathbf{D}_{0} \mathbf{Q}_{t}
\end{aligned}
$$

The first equality follows from the definition of channel $\mathbf{G}$ and the last from the fact that the distribution of $\mathbf{Q}_{t}$ is rotation independent, as previously discussed. By assumption $\mathbf{Q}_{1}, \ldots, \mathbf{Q}_{T}$ are independent then the average BER is

$$
\overline{B E R}(P) \leq \mathbb{E}_{\mathbf{H}, \mathcal{T}}\left[\mathcal{Q}\left(\sqrt{\frac{2 P}{\sigma^{2}} \eta \lambda_{K}^{2}[\mathbf{H}]}\right)\right]
$$

where $\eta=\max _{\mathbf{Q} \in \mathcal{T}} \lambda_{K}\left[\mathbf{Q}^{H} \mathbf{D}_{0}^{H} \mathbf{D}_{0} \mathbf{Q}\right]$ represents the loss incurred by the fact that the equalizer is selected from a finite set.

From (15) we verify that the right and left singular vectors of the channel do not influence the average BER. This happens as the right singular vectors of the channel may be incorporated in the precoders without changing their distribution or their independence properties. From [17] [18] we can lower bound $\lambda_{K}\left[\mathbf{Q}^{H} \mathbf{D}_{0}^{H} \mathbf{D}_{0} \mathbf{Q}\right]$ by

$$
\begin{aligned}
\lambda_{K} & {\left[\mathbf{Q}^{H} \mathbf{D}_{0}^{H} \mathbf{D}_{0} \mathbf{Q}\right] } \\
& =\left|\mathbf{Q}^{H} \mathbf{D}_{0}^{H} \mathbf{D}_{0} \mathbf{Q}\right|\left(\frac{K-1}{\operatorname{tr}\left(\mathbf{Q}^{H} \mathbf{D}_{0}^{H} \mathbf{D}_{0} \mathbf{Q}\right)}\right)^{K-1} \\
& \geq\left|\mathbf{Q}^{H} \mathbf{D}_{0}^{H} \mathbf{D}_{0} \mathbf{Q}\right|\left(\frac{K-1}{K}\right)^{K-1}
\end{aligned}
$$

The inequality follows from the trace inequality

$$
\left.\operatorname{tr}\left(\mathbf{Q}^{H} \mathbf{D}_{0}^{H} \mathbf{D}_{0} \mathbf{Q}\right)\right)=\sum_{k=1}^{K} \sum_{m=1}^{K}|\mathbf{Q}(m, k)|^{2} \leq K
$$

where the last inequality follows from the fact that $\sum_{m=1}^{M}|\mathbf{Q}(m, k)|^{2}=1$ and $|\mathbf{Q}(m, k)|^{2} \geq 0$. As $((K-$ $1) / K)^{K-1}$ is a monotonic decreasing with $K$ we have

$$
\lambda_{K}\left[\mathbf{Q}^{H} \mathbf{D}_{0}^{H} \mathbf{D}_{0} \mathbf{Q}\right] \geq e^{-1}\left|\mathbf{Q}^{H} \mathbf{D}_{0}^{H} \mathbf{D}_{0} \mathbf{Q}\right|
$$

From (18) we verify that apart from a constant the determinant of $\mathbf{Q}^{H} \mathbf{D}_{0}^{H} \mathbf{D}_{0} \mathbf{Q}$ is enough to lower bound the corresponding minimum singular value. As a consequence the system performance can be upper bounded by

$$
\overline{B E R}(P) \leq \mathbb{E}_{\mathbf{H}, \gamma}\left[\mathcal{Q}\left(\sqrt{\frac{2 P}{e \sigma^{2}} \gamma \lambda_{K}^{2}[\mathbf{H}]}\right)\right]
$$

where $\gamma=\max _{\mathbf{Q} \in \mathcal{T}}\left|\mathbf{Q}^{H} \mathbf{D}_{0}^{H} \mathbf{D}_{0} \mathbf{Q}\right|$. Using the exponential upper bound of the Q-function $\left(Q(x) \leq 1 / 2 e^{-x^{2} / 2}\right)$ and evaluating the expectation operator of (19) over $\lambda_{K}[\mathbf{H}]$ we get (see [19] equations (127) and (128))

$$
\overline{B E R}(P) \dot{\leq} \mathbb{E}_{\gamma}\left[\gamma^{-(M-K+1)}\right]\left(\frac{P}{\sigma^{2}}\right)^{-(M-K+1)}
$$

As a consequence if the expectation $\mathbb{E}_{\gamma}\left[\gamma^{-(M-K+1)}\right]$ is finite we achieve diversity $M-K+1$, which is the diversity achieved when the transmitter has CSIT [15]. In the following we will analyse this term.

Let $s_{t}=\left|\mathbf{Q}_{t}^{H} \mathbf{D}_{0}^{H} \mathbf{D}_{0} \mathbf{Q}_{t}\right|$, for all $t \in\{1, \ldots, T\}$ be a set of independent and identically distributed random variables. After some manipulations it is possible to show that the variable $s_{t}$ can be written as $s_{t}=\prod_{i=1}^{K} r_{i}$, where the random variables $r_{i}$ are independent and have the following probability distribution

$$
f_{R_{i}}\left(r_{i}\right)=a_{i}\left(1-r_{i}\right)^{M-K-1} r_{i}^{i-1}, \quad r_{i} \in[0,1]
$$

with $a_{i}=\frac{\prod_{n=0}^{i-1}(M-K+n)}{(i-1) !}$. From the previous equation, it can be shown that the probability density function (pdf) of random variable $s_{t}$ is upper bounded by a constant $a=\prod_{i=1}^{K} a_{i}$, i.e. $f_{S_{t}}\left(s_{t}\right) \leq a$. Then we have that the pdf of random variable $\gamma$ is upper bounded by

$$
f_{\Gamma}(\gamma) \leq a^{T} T \gamma^{T-1}
$$

Therefore, for $T>M-K+1$ the expectation operator of equation (20) is finite

$$
\mathbb{E}_{\gamma}\left[\gamma^{-(M-K+1)}\right] \leq \frac{a^{T} T}{T-(M-K+1)}
$$

For $T \leq M-K+1$ the expectation operator is not finite. From (20) and (23) we find that for codebook sizes higher or equal than $M-K+2$ there is no loss due to quantization, as the achievable diversity is equal to $M-K+1$.

\section{NumericAl Results}

In this section we present simulation results to verify the sufficient conditions presented in the previous section. The results presented are a function of the bit error rate over the energy per bit to noise power spectral density ratio per antenna $\left(E_{b} / N_{0}\right)$. To estimate the BER for different $E_{b} / N_{0}$ values the Multicanonical Monte Carlo algorithm [20] has been used. The best element from codebook $\mathcal{T}$ is selected using the BER as a metric. A QPSK constellation under Gray mapping rule is considered. 


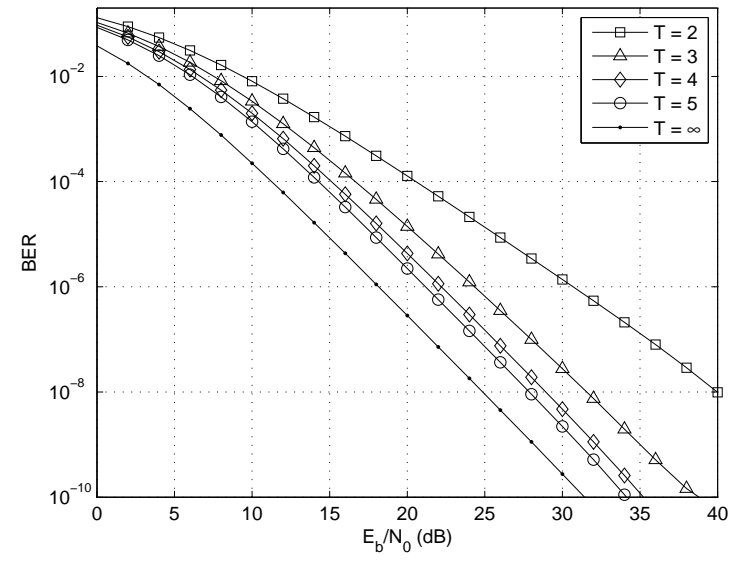

Fig. 2. Average BER for $M=4, K=2$ and $T \in\{2,3,4,5, \infty\}$.

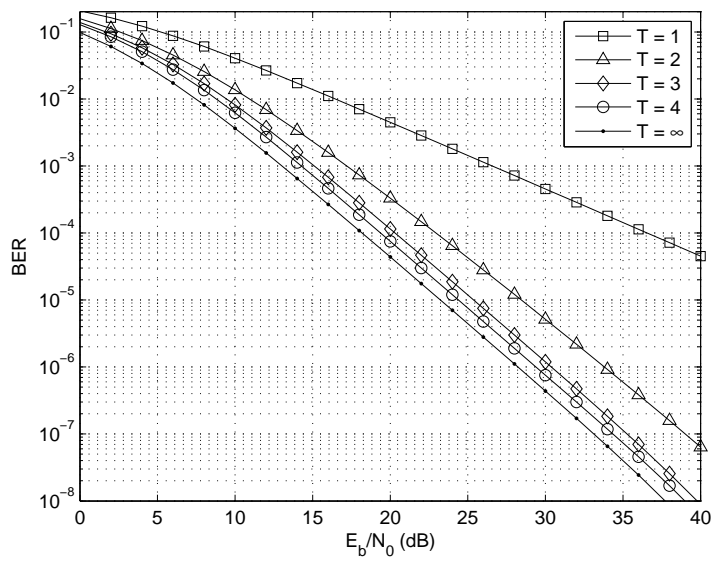

Fig. 3. Average BER for $M=4, K=3$ and $T \in\{1,2,3,4, \infty\}$.

We consider two scenarios, one with $M=4$ and $K=2$ and another with $M=4$ and $K=3$. For both scenarios we present results for different codebook sizes and also for the case where the transmitter has full channel information $(T=\infty)$. In Fig. 2 we present results for the first case. For this scenario the diversity achieved when $(T=\infty)$ is 3 therefore from the previous analysis we need a codebook with size equal or higher than 4 . Indeed, we can verify from this figure that for $T \geq 4$ the slope of the BER curve for high SNRs (the diversity order) is the same. Let us now consider the second scenario. For this case the diversity achieved when $(T=\infty)$ is 2 then the codebook size must be equal or higher than 3 to achieve the same diversity order. In Fig. 3 we present results for the second case. We verify from this figure that for codebooks with size equal or higher than 3 the slope of the BER curves is the same. For lower values of $T$ the diversity achieved is lower as can be attested by Fig. 3. The results presented confirm the derivation made in the previous section.

\section{CONCLUSION}

In this manuscript we have considered a precoded spatial multiplexing system with a ZF receiver. It was assumed that the receiver has accurate channel state information, but the only channel information available at the transmitter is provided by the receiver, through a low rate feedback link. Namely, the receiver quantizes the channel using a codebook known at both terminals, and feeds back the selected index to the transmitter. We have demonstrated that as long as the codebook size is larger than the allowable maximum diversity order then the quantization of the channel leads to no loss in diversity. Indeed, we have shown that to achieve a diversity order of $(D=M-K+1)$ which is the maximum allowed, a codebook with size $1+D$ is enough.

\section{REFERENCES}

[1] E. Telatar, "Capacity of Multi-Antenna Gaussian Channels," European Transactions on Telecommunications, vol. 10, no. 6, pp. 585-595, 1999.

[2] L. Zheng and D. Tse, "Diversity and Multiplexing: A Fundamental Tradeoff in Multiple-Antenna Channels," IEEE Trans. Inf. Theory, vol. 49, no. 5, pp. 1073-1096, 2003.

[3] B. Mondal and R. W. Heath Jr, "A Diversity Guarantee and SNR Performance for Unitary Limited Feedback MIMO Systems," EURASIP Journal on Advances in Signal Processing, vol. 2008, p. 77, 2008.

[4] K. K. Mukkavilli, A. Sabharwal, E. Erkip, and B. Aazhang, "On Beamforming with Finite Rate Feedback in Multiple-Antenna Systems," IEEE Trans. Inf. Theory, vol. 49, no. 10, pp. 2562-2579, 2003.

[5] D. J. Love, R. W. Heath Jr, and T. Strohmer, "Grassmannian Beamforming for Multiple-Input Multiple-Output Wireless Systems," IEEE Trans. Inf. Theory, vol. 49, no. 10, pp. 2735-2747, 2003.

[6] W. Santipach and M. Honig, "Asymptotic Performance of MIMO Wireless Channels with Limited Feedback," Proc. IEEE Mil. Comm. Conf., vol. 1, 2003, pp. 141-146

[7] D. Love and R. Heath, "Equal Gain Transmission in Multiple-Input Multiple-Output Wireless Systems," IEEE Trans. Commun., vol. 51, no. 7, pp. 1102-1110, 2003.

[8] B. Mondal, R. Samanta, and R. Heath, "Frame Theoretic Quantization for Limited Feedback MIMO Beamforming Systems," Proc. IEEE Wireless Networks, Communications and Mobile Computing (IWCMC), 2005, vol. 2, pp. 1065-1070 vol.2.

[9] D. Love and R. Heath, "Necessary and Sufficient Conditions for Full Diversity Order in Correlated Rayleigh Fading Beamforming and Combining Systems," IEEE Trans. Wireless Commun., vol. 4, no. 1, pp. 20 23, 2005 .

[10] P. Zhu, L. Tang, Y. Wang and X. You, "An Upper Bound on the SER of Transmit Beamforming in Correlated Rayleigh Fading," IEEE Trans. Comm. , vol.58, no.2, pp.457,462, February 2010

[11] L. Tang, X. Zhang, Y. Bai and P. Zhu, "Performance Analysis of MIMO Beamforming with Imperfect Feedback." International Journal of Communication Systems, 2012

[12] J. Jalden and B. Ottersten, "On the Maximal Diversity Order of Spatial Multiplexing with Transmit Antenna Selection,” IEEE Trans. Inf. Theory, vol. 53, no. 11, pp. 4273-4276, 2007.

[13] L. Zheng and D. Tse, "Diversity and Multiplexing: A Fundamental Tradeoff in Multiple-Antenna Channels," IEEE Trans. Inf. Theory, vol. 49, no. 5, pp. 1073-1096, 2003.

[14] C. K. Au-Yeung and D. J. Love, "On the Performance of Random Vector Quantization Limited Feedback Beamforming in a MISO System," IEEE Trans. Wireless Commun., vol. 6, no. 2, pp. 458-462, 2007.

[15] E. Sengul, E. Akay, and E. Ayanoglu, "Diversity Analysis of Single and Multiple Beamforming," IEEE Trans. Commun., vol. 54, no. 6, pp. 990-993, 2006.

[16] R. A. Horn and C. R. Johnson, Matrix Analysis. Cambridge University press, 2012.

[17] Y. Yi-Sheng and G. Dun-he, "A Note on a Lower Bound for the Smallest Singular Value," Linear Algebra and its Applications, vol. 253, pp. 25 - 38, 1997.

[18] Q. Zhong and T.-Z. Huang, "Bounds for the Extreme Eigenvalues using the Trace and Determinant," Journal of Information and Computing Science, vol. 3, no. 2, pp. 118-124, 2008.

[19] L. Garcia-Ordonez, A. Pages-Zamora, and J. Fonollosa, "Diversity and Multiplexing tradeoff of Spatial Multiplexing MIMO Systems with CSI," IEEE Trans. Inf. Theory, vol. 54, no. 7, pp. 2959-2975, 2008.

[20] A. Bononi , L. A. Rusch , A. Ghazisaeidi , F. Vacondio and N. Rossi "A Fresh Look at Multicanonical Monte Carlo from a Telecom Perspective", in Proc. IEEE Commun. Soc. GLOBECOM, 2009 